\title{
Elexacaftor/tezacaftor/ivacaftor in an individual with cystic fibrosis caused by a N1303K CFTR variant and infected with Mycobacterium abscessus
}

\author{
E. Elson ${ }^{1}$, Paula Capel $^{1}$, Jessica Haynes ${ }^{1}$, Stephanie Duehlmeyer ${ }^{1}$, Michelle Fischer ${ }^{1}$, and \\ Hugo Escobar ${ }^{2}$ \\ ${ }^{1}$ Children's Mercy Hospital \\ ${ }^{2}$ Childrens Mercy Hospital
}

July 6, 2021

\begin{abstract}
This report describes a case of a 15-year-old male with cystic fibrosis caused by N1303K and Q493X cystic fibrosis transmembrane conductance regulator (CFTR) protein variants. In this case, CFTR modulators including tezacaftor/ivacaftor and subsequently elexacaftor/tezacaftor/ivacaftor were utilized and resulted in clinical stability and improvement.

CFTR MODULATOR THERAPY IN AN INDIVIDUAL WITH CYSTIC FIBROSIS CAUSED BY A N1303K CFTR VARIANT AND INFECTED WITH MYCOBACTERIUM ABSCESSUS

E. Claire Elson, PharmD, BCPPS ${ }^{1}$; Paula Capel, RN ${ }^{1}$; Jessica Haynes, $\mathrm{RN}^{1}$; Stephanie Duehlmeyer, PharmD, BCPPS ${ }^{1}$; Michelle Fischer, $\mathrm{RN}^{1}$; Hugo Escobar, $\mathrm{MD}^{1}$

${ }^{1}$ Children's Mercy Kansas City, 2401 Gillham Rd. Kansas City, MO 64108

Corresponding Author: E. Claire Elson, PharmD, BCPPS

2401 Gillham Rd. Kansas City, MO 64108

816-302-3368 (telephone)

816-302-9736 (fax)

ecelson@cmh.edu

Running Title: CFTR Modulator and N1303K

Key Words: Cystic Fibrosis, CFTR, CFTR Modulator

\section{Introduction}

The utilization of cystic fibrosis (CF) transmembrane conductance regulator (CFTR) modulators has expanded in the last decade. CFTR modulators target the underlying defect of CF. Classified as potentiators or corrector molecules, these medications work independently or in tandem to modify the CFTR protein and improve chloride transport. This improved CFTR function results in increased lung function, decreased pulmonary exacerbations, improved growth and quality of life. In 2019, elexacaftor/tezacaftor/ivacaftor (ETI) was approved for individuals with CF and at least one F508del CFTR variant. As labeling has expanded, now more than $90 \%$ of individuals with CF will qualify for CFTR modulator therapy. ${ }^{1}$
\end{abstract}


For those that do not qualify for CFTR modulator treatment based on their CF variants, other treatments to repair, restore, and fix or replace the CFTR protein continue to be evaluated. In addition to alternative treatment strategies, n-of-1 studies have been completed to demonstrate in vitro and in vivo responses including changes in sweat chloride concentration and improvement in pulmonary function testing. ${ }^{2,3}$

In this case report, we describe a 15-year-old male with cystic fibrosis heterozygous for a N1303K (class II variant) and Q493X (class I variant) receiving ETI.

\section{Case Report}

TN is a 15-year-old male diagnosed with CF in infancy secondary to meconium ileus and CF genetic analysis demonstrating the presence of N1303K and Q493X CF genetic variants. TN had mild CF lung disease and pancreatic insufficiency. He was prescribed respiratory and oral medications as recommended by CF Foundation $(\mathrm{CFF})$ guidelines including bronchodilator, mucolytics with airway clearance, oral pancreatic enzyme replacement therapy, and fat-soluble vitamin supplementation. However, in 2016, TN began to culture nontuberculous mycobacterium (NTM), Mycobacterium abscessus. Despite aggressive airway clearance and treatment of his other chronic infections of methicillin-susceptibleStaphylococcus aureus and Stenotrophomonasmaltophilia, he experienced a slow decline in pulmonary function testing, increased symptoms, and changes on CT scan imaging. Therefore, in late 2016, TN initiated the intensification phase of NTM treatment per the CFF and European Cystic Fibrosis Society guidelines. ${ }^{4}$ Despite aggressive management with four to five intravenous, oral, and inhaled antimicrobials, TN continued to intermittently be smear and culture positive for his NTM infection.

In 2019, based on in vitro evidence of benefit for the N1303K variant and potential modification of CF microbiome with previous CFTR modulators, the CF care team decided to pursue off-label approval of ivacaftor/tezacaftor (IVA/TEZ). ${ }^{5,6}$ TN began treatment with IVA/TEZ in February 2019. The initiation of IVA/TEZ resulted in stabilization of TN's clinical status including lung function parameters, growth trends, and improved quality of life indicated by Cystic Fibrosis Questionnaire Revised (CFQ-R). Repeat sweat chloride testing was completed after one year of treatment with IVA/TEZ and results remained elevated at $115 \mathrm{mmol} / \mathrm{L}$ and $110 \mathrm{mmol} / \mathrm{L}$, which was consistent with the most recent testing done in 2014; $100 \mathrm{mmol} / \mathrm{L}$ and $97 \mathrm{mmol} / \mathrm{L}$. Primarily based on patient reported improvement in quality of life and stabilization of clinical outcome measures, TN was continued on IVA/TEZ.

ETI was approved in 2019 and in vitro evidence of some improvement in CFTR protein function in individuals with N1303K, the CF care team decided to pursue off-label coverage for this treatment. ETI was approved by insurance, and TN transitioned to this treatment in January 2021. Two weeks after ETI therapy initiation, $\mathrm{TN}$ presented to $\mathrm{CF}$ clinic for a standard follow up visit. There were no other changes to his medication regimen. From his previous clinic visit 6 weeks prior, his weight increased $2.9 \mathrm{~kg}$ ( $10^{\text {th }}$ up to $42^{\text {nd }}$ percentile) and his BMI improved from the $29^{\text {th }}$ to the $38^{\text {th }}$ percentile. His lung function improved from $2.98 \mathrm{~L}(84.3 \%$ predicted FEV1) to $3.41 \mathrm{~L}(93.3 \%$ predicted FEV1) and from $3.85 \mathrm{~L}(93.7 \%$ predicted FVC) to $4.20 \mathrm{~L}$ (99\% predicted FVC). TN reported some increased cough and mucus production during the first week of treatment with ETI that resolved without intervention and otherwise felt a subjective improvement in his exercise tolerance. TN was unable to produce expectorated sputum therefore the $\mathrm{CF}$ care team was unable to assess for the presence of NTM infection, but plan to continue to monitor. In addition, TN completed a CFQ$\mathrm{R}$ prior to initiation of ETI and within 12 weeks of being on treatment which demonstrated improvements in the physical, vitality and health domains. Interestingly, his repeat sweat chloride two months after initiation of ETI remained elevated at $113 \mathrm{mmol} / \mathrm{L}$ and $111 \mathrm{mmol} / \mathrm{L}$. At his clinic visit around the same time, he had stable lung function (FEV1 3.39 90.2\%), inability to produce sputum and normal flora on oropharyngeal swab, and increased weight up to 56.2 kilograms ( $43^{\text {rd }}$ percentile for his BMI).

For safety monitoring, TN received annual ophthalmology exams and routine hepatic function monitoring. He had intermittent increased transaminases while on IVA/TEZ that was believed to be secondary to his NTM regimen. He had hepatic function testing prior to initiation of ETI that was within normal limits. A repeat hepatic function panel was done 8 weeks after initiation that showed a mild increase in transaminases 
but less than two times the upper limit of normal. These will continue to be monitored. TN reported no other adverse reactions with initiation of ETI.

\section{Discussion}

TN's genetic variants (Q493X and N1303K) are classified as class I and class II variants, respectively. The Q493X variant is a nonsense variant resulting in decreased quantity of CFTR. The N1303K variant is a class II missense variant with decreased quantity and function of CFTR and is in the same class as the most common CFTR variant, F508del. Individuals with one copy of F508del qualify for ETI treatment. In 2018, an in vitro study demonstrated 8-time improvement in the open probability with ivacaftor and 3-time improvement in biogenesis with corrector molecules (tezacaftor, lumacaftor). ${ }^{5}$ A more recent study in 2020 , found improvement in CFTR function in two individuals homozygous for N1303K and found a significant improvement in CFTR function from nasal epithelial cells within 48 hours of treatment. ${ }^{7}$ The authors did note there did not appear to be a significant change in CFTR processing and theorized the benefit was primarily related to potentiation of membrane N1303K proteins. 7 Other in vitro studies for homozygous N1303K variant found improvements in processing of CFTR. ${ }^{8}$ These data from in vitro analysis are consistent with our case report demonstrating significant clinical benefit in an individual heterozygous for the N1303K variant regarding pulmonary function, growth parameters, and quality of life scores. This therapy was well tolerated with mild increases in hepatic function testing. It is worthwhile to note that despite these clinical parameters for improvement, TN's sweat chloride values remained unchanged with both IVA/TEZ and ETI treatment. This case further demonstrates the importance of n-of-1 study in individuals with $\mathrm{CF}$ and rare variants to ensure all patients that could benefit from CFTR modulator therapy may receive treatment.

\section{References}

1. Gramegna A, Contarini M, Aliberti S, Casciaro R, Blasi F, Castellani C. From Ivacaftor to Triple Combination: A Systematic Review of Efficacy and Safety of CFTR Modulators in People with Cystic Fibrosis. Int J Mol Sci. 2020 Aug 16;21(16):5882. doi: 10.3390/ijms21165882. PMID: 32824306; PMCID: PMC7461566.

2. McGarry ME, Illek B, Ly NP, Zlock L, Olshansky S, Moreno C, Finkbeiner WE, Nielson DW. In vivo and in vitro ivacaftor response in cystic fibrosis patients with residual CFTR function: N-of-1 studies. Pediatr Pulmonol. 2017 Apr;52(4):472-479. doi: 10.1002/ppul.23659. Epub 2017 Jan 9. PMID: 28068001 ; PMCID: PMC5461115.

3. Nick JA, St Clair C, Jones MC, Lan L, Higgins M; VX12-770-113 Study Team. Ivacaftor in cystic fibrosis with residual function: Lung function results from an N-of-1 study. J Cyst Fibros. 2020 Jan;19(1):91-98. doi: 10.1016/j.jcf.2019.09.013. Epub 2019 Nov 26. PMID: 31784217.

4. Floto RA, Olivier KN, Saiman L, Daley CL, Herrmann JL, Nick JA, Noone PG, Bilton D, Corris P, Gibson RL, Hempstead SE, Koetz K, Sabadosa KA, Sermet-Gaudelus I, Smyth AR, van Ingen J, Wallace RJ, Winthrop KL, Marshall BC, Haworth CS; US Cystic Fibrosis Foundation and European Cystic Fibrosis Society. US Cystic Fibrosis Foundation and European Cystic Fibrosis Society consensus recommendations for the management of non-tuberculous mycobacteria in individuals with cystic fibrosis. Thorax. 2016 Jan;71 Suppl 1(Suppl 1):i1-22. doi: 10.1136/thoraxjnl-2015-207360. PMID: 26666259 ; PMCID: PMC4717371.

5. DeStefano S, Gees M, Hwang TC. Physiological and pharmacological characterization of the N1303K mutant CFTR. J Cyst Fibros. 2018 Sep;17(5):573-581. doi: 10.1016/j.jcf.2018.05.011. Epub 2018 Jun 7. PMID: 29887518; PMCID: PMC7008954.

6. Rowe SM, Heltshe SL, Gonska T, Donaldson SH, Borowitz D, Gelfond D, Sagel SD, Khan U, MayerHamblett N, Van Dalfsen JM, et al .; GOAL Investigators of the Cystic Fibrosis Foundation Therapeutics Development Network. Clinical mechanism of the cystic fibrosis transmembrane conductance regulator potentiator ivacaftor in G551D-mediated cystic fibrosis. Am J Respir Crit Care Med2014;190:175184

7. Laselva O, Bartlett C, Gunawardena TNA, et al. Rescue of multiple class II CFTR mutations by elexacaftor+ tezacaftor+ivacaftor mediated in part by the dual activities of Elexacaftor as both corrector 
and potentiator. Eur Respir J 2020; in press.

8. Veit G, Roldan A, Hancock MA, Da Fonte DF, Xu H, Hussein M, Frenkiel S, Matouk E, Velkov T, Lukacs GL. Allosteric folding correction of F508del and rare CFTR mutants by elexacaftor-tezacaftorivacaftor (Trikafta) combination. JCI Insight. 2020 Sep 17;5(18):e139983.

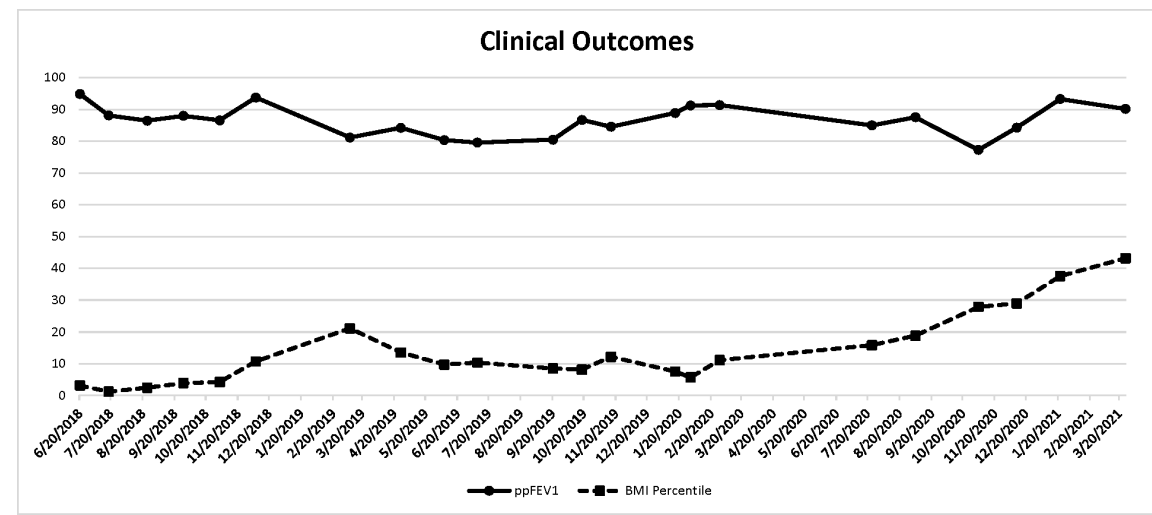

